\title{
DEMO: Eva, um mascote virtual em Realidade Aumentada
}

\author{
Afonso Costa, Rachel Lima, Sergio Tamayo \\ SIDIA - Instituto de Ciência e Tecnologia \\ Manaus, Brasil \\ \{afonso.costa, rachel.lima, sergio.tamayo\}@ sidia.com
}

\begin{abstract}
Resumo-As aplicações de realidade aumentada estão presentes no nosso cotidiano e os telefones inteligentes são atualmente a ferramenta comum que faz isso possível. $O$ principal objetivo deste demo é apresentar uma aplicação de código aberto chamada Eva, a qual é um mascote virtual que vive no mundo de realidade aumentada. Esta aplicação está sendo desenvolvida para dispositivos Android e suas principais funcionalidades são apresentadas neste trabalho.
\end{abstract}

Palavras-chave: realidade aumentada, dispositivos móveis, mascote virtual, entretenimento

\section{INTRODUÇÃO}

A Realidade Aumentada (RA) está sendo utilizada em diversas áreas [1]-[3] e, principalmente, no entretenimento [4]-[6], onde as previsões preveem ser um mercado crescente [7]-[9], e onde os dispositivos móveis (principalmente os smartphones) têm sido bastante utilizados como ferramentas que permitem aos usuários interagirem com objetos virtuais inseridos em um ambiente real. Jogos como AR Dragon e Jurassic World destacam-se nessa emocionante interação digital. Dentre o conjunto destas soluções encontram-se os mascotes virtuais, os quais são utilizados principalmente por crianças, ensinando-as de forma divertida a responsabilidade de cuidar de um animal de estimação virtual.

Neste contexto de RA, jogos digitais para crianças e imersão digital, apresenta-se a mascote virtual Eva, aplicativo para smartphones compatíveis com o sistema Android a partir da versão 8. Este aplicativo é gratuito e Open Source [10], destacado por sua interação e a possibilidade de compartilhar momentos de imersão.

O objetivo principal deste demo foca em apresentar as principais funcionalidades do aplicativo, tais como: compartilhamento de cena e de momentos de imersão, animações e simulações de física no ambiente. Estas funcionalidades são descritas nas próximas seções.

\section{ObJetivo}

Estudos têm indicado que a relação com os jogos digitais, principalmente, relacionadas em atividade direcionadas e orientadas, pode melhorar o controle inibitório em crianças nos anos pré-escolares [11], contribui na ativação da rede de atenção executiva de forma mais rápida e eficiente [12], oferece ganhos significativos no desempenho da memória de trabalho [13] e, claro, contribui para a diversão das crianças.
Dentre os jogos similares em RA com a temática de interação com uma mascote virtual podem-se destacar, o AR Dragon [4], o Meow! - AR Cat [5] e o My Very Hungry Caterpillar [6]. Estas aplicações focam principalmente no objetivo de atrair e manter a atenção dos jogadores mediante a realização de tarefas lúdicas, divertidas e motivadoras, provocando engajamento e aumentando as chances de aprendizado efetivo. Mas na atualidade, outras atividades como o compartilhamento social de atividades que estão sendo executadas ou fatos acontecidos e a realização de tarefas em modo cooperativo e (ou) colaborativo despertam interesse e são o foco deste trabalho. No restante do demo são apresentadas as funcionalidades e potencialidades da mascote virtual Eva.

\section{Desenvolvimento e Resultados}

A Eva é uma aplicação que está sendo construída utilizando o framework Samsung XR (SXR) [14] com suporte ao ARCore [15].

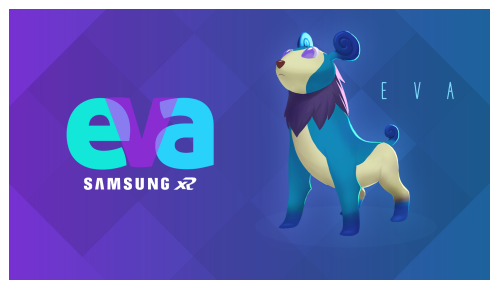

Figura 1. Splash Screen da aplicação Eva

Na Figura 11 pode-se conhecer a mascote virtual Eva no momento que a aplicação é inicializada. Todas as ações e interações possíveis, estão listadas e podem ser visualizadas na tela principal como mostra a Figura 2

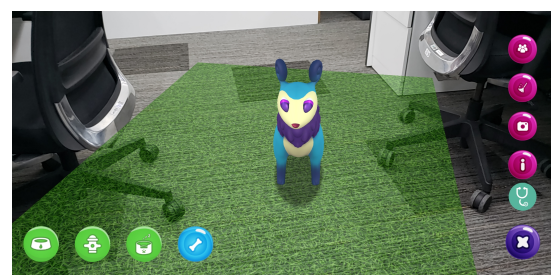

Figura 2. Tela inicial da aplicação

\section{Ações da Eva}

Os usuários poderão interagir com Eva através de um conjunto de ações pré-definidas, dentre as quais destacam-se: 
- Locomoção no plano identificado;

- Interação com objetos virtuais. (Figura 3(a);

- Interação com brinquedo. (Figura 3(b)).

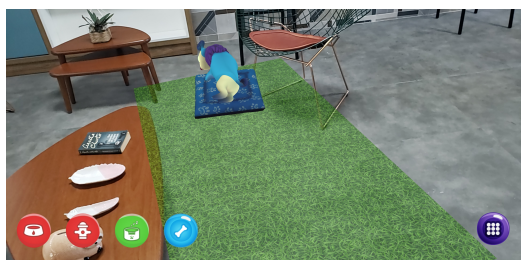

(a) Eva dormindo na cama

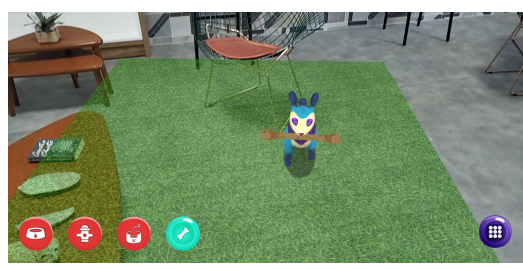

(b) Eva brincando com o osso

Figura 3. Exemplos de ações da mascote em cenários reais

\section{Compartilhamento de Cena e Imagens}

$\mathrm{O}$ usuário pode compartilhar a cena que ele está visualizando no dispositivo com outros usuários que tenham a aplicação instalada e estejam no mesmo ambiente real. Este compartilhamento só é possível devido à:

- Utilização de uma funcionalidade do ARCore chamada de Cloud Anchor, e a;

- Criação e utilização de uma rede Bluetooth entre os dispositivos.

Uma vez que a âncora do objeto 3D está compartilhada entre os dispositivos e a rede Bluetooth está criada, as interações feitas com a Eva no dispositivo servidor serão enviadas via comandos específicos para todos os usuários (dispositivos clientes) que estão imersos no ambiente e distribuídos pela cena (Figura 47.

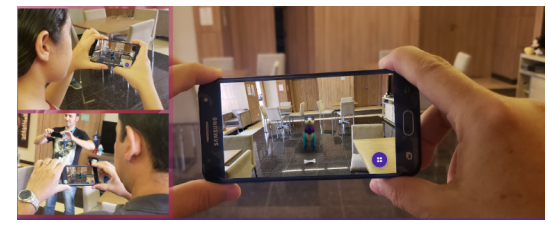

(a) Dispositivos compartilhando a cena

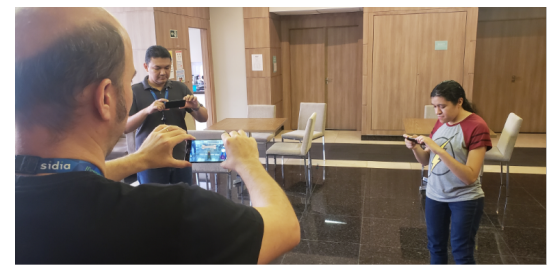

(b) Posicionamento dos usuários

Figura 4. Demonstrando o compartilhamento de cena

Dado o número de usuários que manifestaram o interesse de compartilhar os momentos de imersão e interação com a
Eva, foi adicionada uma funcionalidade para que o usuário possa capturar e compartilhar as imagens nas principais mídias sociais. Na Figura 5 está ilustrada o momento prévio da divulgação de uma imagem.

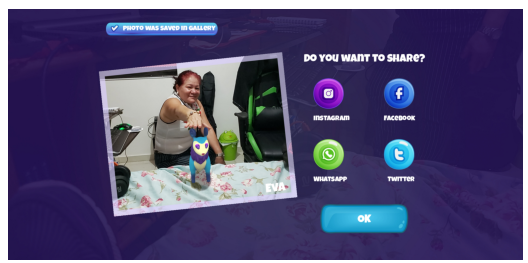

Figura 5. Captura e compartilhamento de imagens em redes sociais

\section{CONSIDERAÇ̃̃Es FinAis}

Apresentou-se um aplicativo de RA para entretenimento de crianças e suas principais funcionalidades de interação, destacando os compartilhamentos de cena e de momentos de imersão (através de divulgação nas mídias sociais) como funcionalidades não presentes em aplicações similares e demandadas pelos usuários.

\section{REFERÊNCIAS}

[1] T. Blum, R. Stauder, E. Euler, and N. Navab, "Superman-like x-ray vision: Towards brain-computer interfaces for medical augmented reality," in 2012 IEEE International Symposium on Mixed and Augmented Reality (ISMAR), Nov 2012, pp. 271-272.

[2] M. Lorenz, S. Knopp, and P. Klimant, "Industrial augmented reality: Requirements for an augmented reality maintenance worker support system," in 2018 IEEE International Symposium on Mixed and Augmented Reality Adjunct (ISMAR-Adjunct), Oct 2018, pp. 151-153.

[3] T. Blum, V. Kleeberger, C. Bichlmeier, and N. Navab, "mirracle: An augmented reality magic mirror system for anatomy education," in 2012 IEEE Virtual Reality Workshops (VRW), March 2012, pp. 115-116.

[4] PlaysideStudios. (2017) Ar dragon. [Online]. Available: https://www. playsidestudios.com/ar-dragon

[5] Innovis. (2017) Meow! - ar cat. [Online]. Available: http://innovis.io/ arcat/

[6] Storytoys.com. (2015) My very hungry caterpillar. [Online]. Available: https://storytoys.com/apps/my-very-hungry-caterpillar/

[7] T. Hennig-Thurau and M. B. Houston, "The fundamentals of entertainment," in Entertainment Science. Springer, 2019, pp. 41-57.

[8] M. J. Douma, Newspapers and knowledge in the market revolution. SAGE Publications: SAGE Business Cases Originals, 2019.

[9] A. Sharma, "3d animation software market size, outlook research report 2018-2025," 2019.

[10] SIDIAVR. (2018) Eva. [Online]. Available: https://github.com/sidia-sxr/ eva

[11] D. M. Healey and J. M. Halperin, "Enhancing neurobehavioral gains with the aid of games and exercise (engage): Initial open trial of a novel early intervention fostering the development of preschoolers' selfregulation," Child Neuropsychology, vol. 21, no. 4, pp. 465-480, 2015.

[12] M. R. Rueda, P. Checa, and L. M. Combita, "Enhanced efficiency of the executive attention network after training in preschool children: immediate changes and effects after two months," Developmental cognitive neuroscience, vol. 2, pp. S192-S204, 2012.

[13] R. Nouchi, Y. Taki, H. Takeuchi, H. Hashizume, T. Nozawa, T. Kambara, A. Sekiguchi, C. M. Miyauchi, Y. Kotozaki, H. Nouchi et al., "Brain training game boosts executive functions, working memory and processing speed in the young adults: a randomized controlled trial," PloS one, vol. 8, no. 2, p. e55518, 2013.

[14] Samsung. (2018) Samsungxr. [Online]. Available: http://www. samsungxr.com/

[15] GoogleARCore. (2018) Arcore. [Online]. Available: https://developers. google.com/ar/develop/ 